\title{
Persuasive technology for overcoming food cravings and improving snack choices
}

\author{
Anne Hsu ${ }^{1}$, Jing Yang ${ }^{1}$, Yigit Yilmaz ${ }^{1}$, Md Sanaul Haque ${ }^{1}$, Cengiz Can ${ }^{1}$, Ann Blandford ${ }^{2}$ \\ Queen Mary, University of London \\ School of Electronic Engineering and Computer Science \\ Mile End Road, London, E14NS \\ \{anne.hsu, j.yang, y.yilmaz, m.haque, c.can\}@qmul.ac.uk \\ University College London \\ UCL Interaction Centre \\ Gower Street, London WC1E 7JG \\ a.blandford@ucl.ac.uk
}

\begin{abstract}
A central challenge in weight management is the difficulty of overcoming desires for excessive and unhealthy food. Yet, studies show that when people are able to resist their desires for unhealthy choices, they experience pride and satisfaction. In order to alleviate the former and support the latter, we designed, implemented and tested a mobile application for improving snacking behavior. Our application delivers a food craving reduction intervention at the moment of need and allows users to track how often they successfully resisted cravings. Our craving reduction intervention is based on recent research that shows that food cravings can be reduced through imagery techniques. We conducted a week-long evaluation of our application, comparing the effectiveness of our application to a basic tracking application. We found that our imagery application significantly reduced both overall snacking and unhealthy snacking compared to a simple snack-tracking application.
\end{abstract}

\section{Author Keywords}

Persuasive technologies; behavior change; weight management; mobile; wellness; user-centered design

\section{ACM Classification Keywords}

H5.m. Information interfaces and presentation (e.g., HCI): Miscellaneous. J.3. Life and medical sciences: Health.

\section{General Terms \\ Human Factors.}

\section{INTRODUCTION}

Persuasive technologies effect change through two primary (non-mutually exclusive) avenues: by targeting either external behaviors or internal attitudes. The overwhelming majority of existing persuasive technologies focus on

\footnotetext{
Permission to make digital or hard copies of all or part of this work for personal or classroom use is granted without fee provided that copies are not made or distributed for profit or commercial advantage and that copies bear this notice and the full citation on the first page. Copyrights for components of this work owned by others than ACM must be honored. Abstracting with credit is permitted. To copy otherwise, or republish, to post on servers or to redistribute to lists, requires prior specific permission and/or a fee. Request permissions from Permissions@acm.org. CHI 2014, April 26 - May 01 2014, Toronto, ON, Canada Copyright $2014 \quad$ ACM $978-1-4503-2473-1 / 14 / 04 \ldots \$ 15.00$. http://dx.doi.org/10.1145/2556288.2557099
}

external behaviors [1], e.g. a tracker for calorie consumption and exercise [15]. This is because external behaviors are more tangible, measurable, and thus easier targets for change. However, the distinction between influencing behavior vs. thought is often blurred: often influencing behavior also promotes new thoughts and awareness. For example exercise and calorie trackers increase user awareness of their activities and intake. Along these lines, there has been a recent increase in persuasive technologies designed for psychological influence by encouraging reflection and awareness about health related choices. These include a visual garden display to encourage emotional connection to one's levels of physical activity [6, 7], open-ended platforms for documenting and sharing health decisions, and a shared visual journal for diet and exercise [8].

It is important for persuasive systems to change attitudes as well as behavior because attitudes and psychological processes are the underpinnings of human choices and behavior [9-11]. Many technologies such as mobile applications are particularly well-positioned to deliver interventions that target psychological processes because they are pervasively by our side. These advantages of pervasive technologies have been summarized through a description of the many roles that such technologies play [12]: a device of 'kairos' that is always near at hand; the role of a concierge that offers guidance and information at the moment of need; a personal coach that can track personal goals and context; and a jester that can promote fun interactions (both personal and social). These properties of technological systems make them well suited for modifying psychology because they can evoke new thought processes and emotions in a timely fashion, customized to the persuasion context. For example, technologies can be synchronized with electronic diaries or sensing technologies [13] to know when contexts that prompt salient psychological states will occur. Finally, thoughts, feelings, and attitudes are highly personal, and technology-based systems have the potential for delivering personalized interventions that cater to this individual variation.

\section{Designing for specific psychological constructs}

In a movement towards more psychology-inspired design, recent persuasive health technologies have started applying 
theories and strategies from psychology and health research $[1,3,1416]$. While knowledge of context-general psychological principles is highly useful for persuasive design, it is also important to understand the specific psychological constructs that underlie the behaviors in a particular design context. Recent work has suggested that context-specific psychological constructs can be usefully investigated from the perspective of a decision theory framework [17]. Decision theory explains how behavioral choices are underpinned by psychological rewards and costs valuations [9]. Behaviors are chosen when they are associated with greater rewards and fewer costs. This notion of rewards and costs is fundamentally a hedonistic concept, relating to emotional experiences of pleasure and displeasure in the brain. An understanding of the rewards and costs associated with healthy and unhealthy behaviors can inform the design of persuasive health technologies: systems can then aim to increase the rewards and decrease the costs associated with healthy behaviors.

A recent study used such a decision theoretic approach to investigate the psychological constructs that discouraged and encouraged weight management behavior [17]. It was found that the need to restrict the pleasure of eating was one of the most widely experienced struggles involved in weight loss. On the other hand, another widely expressed view was that people felt good after resisting food temptations and making healthy choices. People felt pride, and satisfaction associated with being good to oneself and having self control. We wished to design an application that addressed both of these psychological weight loss constructs: to help people with their struggle against food temptations while simultaneously enhancing their ability to feel good about healthy choices. As a method of reducing the difficulty of unhealthy temptations, we look to recent research on the psychology of cravings, which describes both how cravings may be sustained in the mind and how they can be reduced. This is known as the Elaborated Intrusion (EI) Theory of Desire [18-21], which we describe briefly below.

\section{Food Cravings: El Theory of Desire}

The Elaborated Intrusion (EI) Theory of Desire is a theory for how appetitive cravings (including food) are sustained through cognitive processes [18-25]. EI theory suggests that cravings involve a cycle of mental elaboration of an initial intrusive thought. Because these thoughts are initially pleasurable, the individual is motivated to elaborate the thought, and sustain the craving by retrieving cognitive associations and creating mental imagery of the target. These mental imagery elaborations recruit working memory functions and may include the senses of sight, taste, touch, and hearing. Because cravings are thus presumed to be sustained through specific cognitive processes, EI theory proposes that an alternative task that recruits similar mental resources could conversely disrupt the elaboration of desire-related intrusive thoughts and thus reduce cravings.
Consistent with this idea, many studies have found that engaging in visual or olfactory imagery reduces the strength of food cravings, both for cravings induced in the lab $\left[20^{-}\right.$ 22] and naturally occurring 'in the wild' [25]. In contrast, auditory imagery tasks were not found to reduce cravings.

While the above results are very promising, they only measured self-reported decrease in craving levels and effects on consumption were not assessed. Our study will be the first to evaluate whether this method of craving reduction results in reduced consumption 'in the wild'. We designed and implemented a mobile application for improving snacking behavior. Our application is designed to alleviate the struggle for excessive consumption while enabling users to emphasize the times where they successfully made healthy choices by either choosing a healthy snack or resisting a snack entirely. Because previous research has shown that simple tracking is effective for improving eating habits [3], we assessed the effectiveness of our application for improving snacking by comparing its effects to that of a basic tracking application in a one week experimental study. Finally we discuss future directions for improving our application.

\section{SYSTEM DESIGN: ICRAVE}

We designed a technology we call iCrave to help people improve their snacking habits. iCrave is a simple mobile app that allows users to track their snack cravings and allows them to record whether they choose to have a healthy or unhealthy snack, or to forgo a snack. iCrave helps users in the moment of desire for unhealthy/excessive snacking by prompting a craving-reduction imagery task and allowing users to record their subsequent choices.

The usage of iCrave is as follows: When the user experiences a snack craving, they press the main 'iCrave' button. The application then shows a text prompt asking users to imagine a specific passive or active scene for 10 seconds, during which a 10 second countdown timer is shown. There were 20 different suggested visualizations based on those used in previous work, which appeared in block random order $[21,26]$. This type of text-prompted imagery exercise has been shown in previous research to reduce craving intensity [18-24]. Because we wanted to check whether imagination intensity was correlated with effectiveness, and also to emphasize engagement with the visualization task, after the 10 second imagery task, we asked users to rate on a 5 point scale how vividly they imagined the scene, ranging from 'No image at all' to 'perfectly vivid, just like the real thing'. Then users were allowed to make one of three choices on the application, by either not eating at all and therefore 'saving' their craving, or choosing a healthy or unhealthy snack. If the user chooses to not eat at all, they are congratulated on 'saving' their craving and the system keeps track of a 20 


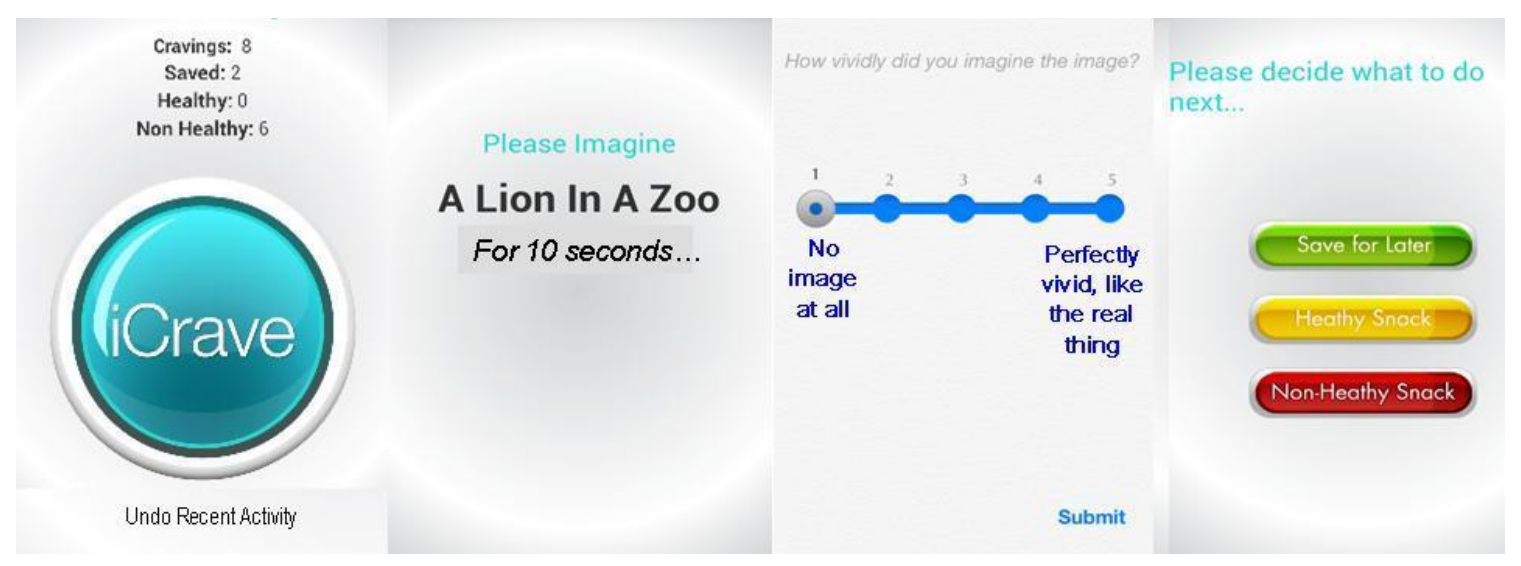

Figure 1. Screen shots of iCrave application

minute period, during which they are not allowed to choose to either eat or save a craving again. A 20 minute period was chosen because research has shown it takes at least 10 minutes for the brain to register fullness after consumption [27] and post-consumption experiences such as happiness and guilt peaked 5-30 minutes after eating [28]. If the user chooses to snack, there is no counter and they are allowed to snack again immediately. On the main screen a counter is shown that tracks total number of events of cravings, savings (no snacking), healthy and unhealthy snacks. We chose this simple level of tracking to minimize user burden while still offering a reasonable level of monitoring. Initial prototype evaluations verified that our design was simple and intuitive to use. An 'undo recent activity' button was provided to allow users to correct mistaken entries or report snacks they might have eaten while on the 20 minutes savings period. See Figure 1 for screen shots of our application. Below we summarize the main principles that inspired the design.

\section{Enhancing rewards of healthy choices}

Typically, when a person feels the temptation to consume excessive/unhealthy foods, there is little reward available for resisting the temptation. Previous work has found that people do feel good about resisting food temptations [17]. However, the moments of feeling good can be diminished as the moment becomes lost in memory. Current applications only track intake, and we do not know of any applications that specifically allow users to record moments where food is avoided. By recording moments of forgoing snacking despite experiencing cravings, we allow users to remember these moments of successful self control. Because we wanted to encourage the idea that forgoing immediate rewards should allow for greater rewards later, we called this event a 'saving' of the craving on the app.

\section{Just in time intervention}

One of the many heralded advantages of mobile applications is their ever-present nature, which allows them to deliver support at the moment of need. Along these lines,
iCrave functions like an emergency aid response. When a snack craving hits, the user can use our application to obtain a 'just in time' delivery of our intervention. Thus our application operates at the key moment of necessity. While application-initiated interventions are also useful for weight loss, e.g., reminders and prompts, we feel it is also important to create applications where the onus is on the user to initiate to initiate engagement in order to promote user awareness. Thus our application was designed to enhance individuals' awareness of their snack cravings and promote a sense of agency in the user's efforts towards self control.

\section{LITE PROTOTYPE EVALUATION}

Before we implemented our full application, we tested general usability of our design on a 'lite' prototype. This 'lite' prototype allowed users to track craving savings and healthy vs. unhealthy snacks, but did not prompt the craving-reducing imagery task. The aim of our prototype pilot was to evaluate: whether the interface was intuitive to use; whether people would readily use the app during snack cravings; and whether it felt intuitive to use a 'save' button if the user chose to forgo snacking. As with our full application, if the user chose to 'save' their snack craving, they would not be able to use the application for 20 minutes. The prototype was deployed on a mobile-friendly web application so that it could be used on all mobile platforms. Our prototype did not store user data onto a database; individual user data (snack counts etc.) were stored in cookies in the individual's web browsers. We recruited 23 participants from the local community; all were healthy adults who had an interest in improving their snacking habits. They used our prototype app over a weeklong period. Post-questionnaires verified that people were willing to use the app when they experienced a craving, and readily used the 'save' button when they felt they could forgo the snack. We also interviewed 13 participants and asked them about their experience using the app. Even when using just the lite prototype version, most felt the app helped them make healthier choices. "It slowed down my 
rate of taking unhealthy foods and also motivated me." "After using this app I become cautious of taking junk food, started avoiding junk food." "I use the iCrave app to remind myself that I should control my appetite." To evaluate usability, we asked how easy/hard they felt the app was to use. They all reported it was very usable. 'Very easy', 'simple', 'convenient', 'very user friendly, I like it very much'. The main complaints were all centered on the fact that it wasn't deployed as an app and only as a mobile site. Given the positive qualitative results and positive survey results, we concluded that our prototype evaluation verified the basic usability of our design and warranted the implementation of our full application.

\section{MAIN EVALUATION STUDY}

Our full application extended the prototype version with the following additional features: When the iCrave button was pressed, the app prompts a 10s imagery task for craving reduction followed by a request for the user's vividness rating for the imagery task. We also implemented a simpler snack-tracker version for comparison purposes. We evaluated the effectiveness of our full iCrave application by comparing its effectiveness with the snack-tracker in a oneweek between-subjects study. The snack tracker was almost identical to the full iCrave app, except that it did not prompt an imagery task or vividness rating, and did not provide the choice of a 'savings' button to record the forgoing of a snack. Thus, the snack tracker looked exactly like Figure 1 except that the two center screens were omitted, the 'save for later' button was not available in the right screen, and the 'saved' tally was not available on the home screen. Both the full iCrave and snack tracker apps were implemented on both iOS and Android platforms and recorded all user data on a secure database ${ }^{1}$. Participants were randomly assigned to one of two conditions. In the experimental condition, participants used our iCrave application during the week. In the control condition, participants used the snack tracker app. Participants in both conditions were asked to use the app when they experienced a snack craving and indicate whether they were going to consume a healthy or unhealthy snack before eating. Thus, any biases from reporting consumption beforehand would have applied to both groups.

\section{Participants}

48 participants were recruited from the local community through email lists at the university. Participants were recruited by asking for individuals who had an interest in improving their snacking habits. Participants were then randomly assigned to either condition (Experimental

\footnotetext{
${ }^{1}$ Both versions of the app were made available via the relevant app stores for the duration of the study. They should not be confused with https://sites.google.com/site/icraveapp/, which is a different app with a similar name.
}

Condition: $\mathrm{N}=26,10$ females; Control Conditon: $\mathrm{N}=27,8$ females). There were 18 iOS users in each of the experimental and control conditions. The rest used the Android application. All participants were between 20-34 years of age except one who was 53. Participants were healthy adults over the age of 18 . They were paid $£ 7$ each to participate in our study.

\section{Procedure}

Before the start of the experiment, participants were given a consent form along with a brief overview of the application and a short paragraph explaining standard portion sizes for common healthy and unhealthy snacks. Participants were provided a brief introduction to the app for their condition, explaining that it would allow them to track their consumption of healthy and unhealthy snacks. Participants in the experimental condition were also told that they could 'save' their cravings by resisting a snack altogether. All participants were then asked to fill out responses to a prestudy survey, which asked them to rate on a 7 point Likert scale, their motivation to snack more healthily, confidence in ability to snack healthily, likelihood of eating unhealthy snacks without thinking, strength of cravings for unhealthy snacks and snacks overall, ease of resisting unhealthy snacks, and the average number of unhealthy snacks and overall snacks they consumed a day. Participants were then instructed to use their app for the next week to try to improve snacking. At the end of the week, participants filled out a post-study survey asking them again to rate strength of cravings for unhealthy snacks and snacks overall, likelihood of eating unhealthy snacks without thinking, ease of resisting unhealthy snacks and the average amount of snacks they consumed per day the previous week. They were also asked to rate on a 7 point Likert scale how well they did in overcoming unhealthy snack habits during the week of the experiment compared to before.

\section{Measurement}

The main outcome variables of our experiment were the number of healthy, unhealthy, and overall snacks consumed in the two conditions. These values were stored in a database for each user (stored using an anonymous user id). As with our pilot study, we also compared post vs. pre experiment survey answers between the two conditions for questions that were asked both before and after the experiment. Finally we assessed whether vividness of imagery affects the snack choice. Post-experiment qualitative interviews were also conducted with seven participants who used the full iCrave app.

\section{Quantitative Results}

\section{Pre-Study comparison of conditions}

Based on our pre-study questionnaire, we verified that there were no significant differences between the participants in our two conditions: One-way ANOVA's showed there were no pre-experimental differences in BMI between our experimental $(M=21.7 ; S T D=2.9)$ and control $(M=20.5$; 
$\mathrm{STD}=2.8$ ) conditions $\mathrm{p}=.2$. There also were no significant differences in age $(\mathrm{p}=.48)$, motivation to improve snacking $(\mathrm{p}=.53)$, confidence in ability to improve snacking $(\mathrm{p}=.60)$, tendency to engage in unhealthy snacking without thinking $(\mathrm{p}=.57)$, cravings for overall snacks $(\mathrm{p}=.43)$ and unhealthy snacks $(\mathrm{p}=.55)$, ability to resist unhealthy snacks $(\mathrm{p}=.40)$, and average daily consumption of snacks overall $(\mathrm{p}=.99)$, and unhealthy snacks $(\mathrm{p}=.79)$. Thus, we conclude that the characteristics of participants in our two conditions were well counter-balanced, enabling a valid comparison of the effects of our iCrave application vs. the snack-tracker.

\section{Post-Study comparison: usage and snacking}

We compared how consumption of healthy and unhealthy snacks, and overall app usage varied over time and between conditions. Overall usage is equivalent to the total snacks consumed on the tracker, and the total snacks plus savings for the full iCrave app. Mean overall usage over the whole week per participant was 7.2 times, STD $=3.4$ for the experimental condition and 8.3 times, $\mathrm{STD}=4.9$ for the control condition. A repeated-measures ANOVA's with fixed effect of time (days into expt) of overall usage found no significant effect of condition, $p=.39$. A significant effect of time was found $F(6,306)=2.71, p=.014$, with marginal interaction of condition $\mathrm{p}=.09$. Visual inspection of usage over time showed that while usage varied over days, overall decrease with time was small. This was confirmed using Ordinary Least Squares Pooled Errors (OLSPE) regression fit to usage data with parameters of condition and time, which revealed a small negative slope for the parameter of time: $-.048, \mathrm{t}(369)=2.8, \mathrm{p}=.005$. OLSPE fits to usage for individual conditions found slope for time $=-.04, \mathrm{t}(180)=2.9, \mathrm{p}=.004$ (experimental) and slope for time $=-.05 \mathrm{t}(187)=1.69, \mathrm{p}=.09$ (control). Thus, we conclude there was no significant difference between conditions in terms of the number of times the apps were used, and decrease in usage over time was small for both conditions.

Next we assessed how unhealthy snack consumption differed between participants in the two conditions, and whether this changed over time. We compared unhealthy snacking across conditions and time. A repeated measures ANOVA applied to unhealthy snack consumption found a significant effect of condition, $\mathrm{F}(1,306)=14.5, \mathrm{p}=.0004$, and no effects of time $\mathrm{p}=.43$, and no significant interaction, $\mathrm{p}=.29$. Thus, we conclude participants in our experimental condition consumed significantly fewer unhealthy snacks compared to the control condition (Experiment: $M=1.65$, $\mathrm{STD}=1.3$ Control: $\mathrm{M}=3.5, \mathrm{STD}=2.8$ ) and this did not significantly differ over time (See Figure 2).

A similar analysis was applied to the consumption of healthy snacks consumed. While control condition participants consumed more healthy snacks, this difference was not significant, $p=.27$. There was a significant effect of time $F(6,306)=2.37, p=.03$, and no interaction, $p=.5$. As was the case for overall usage, visual inspection of trends over time showed that while consumption of healthy snacks varied over days, overall decrease with time was small. An OLSPE regression fit to data with parameters of condition and time revealed a small negative slope for the parameter of time: $-.03, \mathrm{t}(369)=2.0, \mathrm{p}=.05$. Thus, we conclude amounts of healthy snacks consumed did not differ signficantly between conditions. While there was variation in healthy snack consumption with time, the variation did not differ between conditions and the overall decrease over time was small.

We also analyzed overall consumption. We found that participants in the experimental condition consumed significantly fewer snacks overall compared to controls. Experiment: $\mathrm{M}=5.0, \mathrm{STD}=2.8$, Control: $\mathrm{M}=8.5, \mathrm{STD}=5.0$, (See Figure 2). Because we found no significant differences in consumptions of healthy snacks, this decrease in overall snack consumption was driven primarily by the fact that unhealthy snacks were reduced in the experimental condition while consumption of healthy snacks remained the same. A repeated measures ANOVA applied to overall snack consumption found significant effects of condition $\mathrm{F}(1,306)=9.2, \mathrm{p}=.004$, and time, $\mathrm{F}(6,306)=2.36, \mathrm{p}=.03$, with marginal interaction, $p=.092$. As before, OLSPE regression fit to the full data with parameters of condition and time found a small negative slope for time, -.045, $t(369)=2.7$, $\mathrm{p}=.007$. Fits to individual conditions separately found slope for time $=-.039, \mathrm{t}(180)=2.8, \mathrm{p}=.006$ (experimental) and slope for time $=-.05 \mathrm{t}(187)=1.69, \mathrm{p}=.09$, (control).
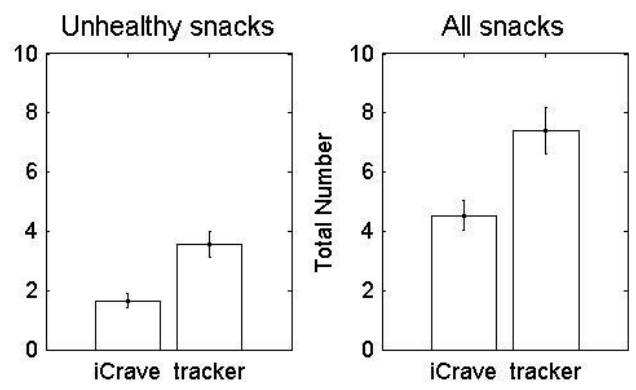

Figure 2. Means and standard errors of total snacks consumed over the week of the experiment. Users of the iCrave application consumed significantly fewer total overall snacks and total unhealthy snacks over a week, compared to users of a simple snack tracker.

Even though the iCrave app and snack tracker were very similar, it is possible that the fact that the hassle of the additional imagery task required by iCrave caused participants in the experimental condition to under-report their snack consumption. Thus, we also asked participants at the end of the study how many unhealthy snacks they consumed over the week. Results suggested participants in both conditions under-reported on the app. However, comparison of unhealthy snack consumption based on postexperiment reports, still supports the main findings that participants in the experimental condition consumed significantly fewer unhealthy snacks, $F(1,51)=4.2, p=0.04$. 
Thus we conclude that our full iCrave app was more effective at reducing unhealthy snack consumption than a basic tracker.

We also checked that our application did not somehow increase the user's amount of snacking compared to the week before the experiment by drawing excessive attention to it. To do this we used t-tests to compare the average number of daily snacks reported in the post-experiment survey to the number reported in the pre-experiment survey $^{2}$. Results analyzed over all participants in both conditions found that participants reported consuming fewer snacks during the week of the study compared to before, $\mathrm{t}(52)=2.3, \mathrm{p}=.03$. When these results were analyzed for each condition separately, we still found decreased snacking: the experimental condition results were near statistical significance, $\mathrm{t}(52)=1.9=.07$, and the control condition results also showed reduced snacking but were not statistically significant. $\mathrm{t}(52)=1.3, \mathrm{p}=.21$. This analysis supports the premise that participants did reduce snacking during the week of the experiment and the iCrave app was more successful at reducing snack consumption through the reduction in unhealthy snacking.

\begin{tabular}{|l|l|l|l|}
\hline & $\begin{array}{l}\text { Mean } \\
\text { rating }\end{array}$ & $\begin{array}{l}\text { STD } \\
\text { rating }\end{array}$ & p-value \\
\hline iCrave & 1.0 & .85 & \multirow{2}{*}{0.02} \\
\hline tracker & .19 & 1.5 & \\
\hline
\end{tabular}

Table 1: Perceived improvement in snacking. Answers were on a 7 point Likert scale of $-3: 3$ corresponding to Much Worse: Much Better

\begin{tabular}{|l|l|l|l|}
\hline & $\begin{array}{l}\text { Mean } \\
\text { rating }\end{array}$ & $\begin{array}{l}\text { STD } \\
\text { rating }\end{array}$ & p-value \\
\hline iCrave & 0.4 & 1.1 & \multirow{2}{*}{0.009} \\
\hline tracker & -.5 & 1.4 & \\
\hline
\end{tabular}

Table 2: Self-reported ease of resisting unhealthy snacks, where answers were on a 7 point Likert scale of -3: 3 corresponding to Extremely Hard: Extremely Easy.

\footnotetext{
${ }^{2} \mathrm{We}$ also repeated analysis of all of our Likert response data assuming ordinal data using the Wilcoxon rank sum test for equal medians. All results and significance values remained virtually the same.
}

Post-Study comparison: survey answers

We conducted one-way ANOVAs comparing experimental vs. control participant's answers to the post-survey regarding how successful participants felt in overcoming unhealthy snacking habits, and how easy they found it to

\begin{tabular}{|l|l|l|l|}
\hline & $\begin{array}{l}\text { Mean } \\
\text { rating }\end{array}$ & $\begin{array}{l}\text { STD } \\
\text { rating }\end{array}$ & p-value \\
\hline iCrave & -.464 & 1.3 & \multirow{2}{*}{0.16} \\
\hline tracker & .11 & 1.5 & \\
\hline
\end{tabular}

Table 3: Self-reported agreement to statement about likelihood eating unhealthy snacks without thinking where answers were on a 7 point Likert scale of -3: 3 corresponding to Completely Disagree: Completely Agree

\begin{tabular}{|l|l|l|l|}
\hline & $\begin{array}{l}\text { Mean } \\
\text { rating }\end{array}$ & $\begin{array}{l}\text { STD } \\
\text { rating }\end{array}$ & p-value \\
\hline iCrave & -.6 & 1.5 & \multirow{2}{*}{0.16} \\
\hline tracker & 0.04 & 1.8 & \\
\hline
\end{tabular}

Table 4: Self-reported agreement to statement about having strong cravings for unhealthy snacks where answers were on a 7 point Likert scale of -3: 3 corresponding to Completely Disagree: Completely Agree

resist unhealthy snacks during the week. Results showed participants in the experimental condition reported significantly greater perceived improvement in overcoming their unhealthy habit compared to controls $(\mathrm{F}(1,51)=5.4$, $\mathrm{p}=.020)$, see Table 1 , and found it easier to resist unhealthy snacks $(\mathrm{F}(1,51)=7.4, \mathrm{p}=.009)$, see Table 2 . While not reaching significance, but with trends in the right direction, experimental participants also reported being less likely to eat unhealthy snacks without thinking $(\mathrm{F}(1,51)=2.1, \mathrm{p}=.16)$, see Table 3, and felt less strong cravings for unhealthy snacks $(F(1,51)=2.1, p=.16)$, see Table 4, compared with control condition participants. Thus users of iCrave felt more successful about improving their snack habits and also felt more able to resist unhealthy snacks compared to users of the tracker.

Pre vs. Post-Study changes in survey answers

Another way of assessing the effects of the application is to compare the changes in answers to questions that were asked both pre and post experiment. Experimental participants showed significant changes in their answers to 
post-study survey questions compared to the same questions in the pre-study survey whereas control participants did not. Paired within-participant t-tests comparing pre vs. post answers for the same participants showed experimental participants after the week had significantly reduced cravings for snacks overall $(\mathrm{t}(25)=5.27, \mathrm{p}=3 \mathrm{e}-5)$ and reduced cravings for unhealthy snacks $(\mathrm{t}(25)=3.1, \mathrm{p}=.005)$, and found it much easier to resist unhealthy snacks $(\mathrm{t}(25)=5.4, \mathrm{p}=.1 \mathrm{e}-5)$. Experimental participants also reported being less likely to consume unhealthy snacks without thinking in the post vs. pre survey, but this effect was not significant $\mathrm{p}=.18$. In contrast, control participants only showed a significant decrease in their reports for cravings for snacks overall, $\mathrm{t}(26)=2.2$, $\mathrm{p}=.04$, and no other changes in post vs. pre study answers were significant.

\section{Imagery vividness and snacking response}

Previous work has shown that the vividness of imagery did not correlate with reductions in cravings [18]. To check whether this lack of correlation existed for craving reduction 'in the wild', we examined for experimental participants whether there were any differences in reported vividness of imagery corresponding to events where participants chose to either save, healthy snack, or unhealthy snack. A one-way ANOVA showed significant differences in vividness ratings across the different snack choices: Save: $\mathrm{M}=3.8, \mathrm{STD}=1.0 ;$ Healthy: $\mathrm{M}=3.8$, STD=.92; Unhealthy: $\mathrm{M}=3.2, \quad \mathrm{STD}=.88 \quad(\mathrm{~F}(1,51)=10.8$, $\mathrm{p}=3 \mathrm{e}-5)$. Thus, we conclude that the vividness to which users were able to engage in the imagery task was related to the effectiveness of the app in improving snack choices.

\section{Qualitative Results}

Post-study interviews were conducted with seven randomly selected participants who participated in the experimental condition and used the full iCrave app for a week. Participants were asked about the external contexts as well as the inner thoughts and feelings that surrounded usage of iCrave. They were asked to describe moments where iCrave prompted them to either forgo a snack or choose a healthy snack, as well as moments where iCrave was not effective.

\section{Moments of resisting snacking entirely}

We asked participants to describe their experiences in instances where use of iCrave helped them resist snacking entirely. Responses supported the idea that cravings for snacks were indeed reduced. Participants reported the reduction in desire for food. "There is a loss of appetite.", "it made me feel like I don't want to eat snacks." "The attraction of snacks is reduced." Participants also described how the imagery task gave them the presence of mind to make better judgments and exert self control about snacking. E.g., "Before I wanted a cookie... afterwards I felt it would be better that I did not eat a cookie." "Afterwards, Ifelt like I could control myself."

\section{Moments of choosing healthy over unhealthy snacks}

We also asked participants what their experiences were when use of iCrave helped them choose a healthy snack. Responses suggested that in these instances, iCrave shifted initial unhealthy preferences towards healthier choices. E.g., "Before, I wanted chocolate immediately. (Afterwards) I preferred the vegetable salad." "It made me prefer healthy food." Similarly to the situations where iCrave heled participants avoid snacks entirely, participants described how iCrave gave them the presence of mind to choose a healthier option: "I wanted chips, but afterwards, I felt it is better to eat something healthy." "After using the app, I wanted to order the healthy snack." A few participants spoke of how iCrave caused them to reflect more generally on the importance of healthy choices: "After using the app, I thought, ice cream is not good for body. I shouldn't just eat it, even when I'm not using the app." "With the app, I became more aware of the food (I chose)."

\section{Feeling good}

All participants reported feeling good after the app helped them either avoid snacking or eat healthier snacks. E.g., "I felt good avoiding the popcorn." "It was a good feeling to avoid the ice cream". "I felt happy to get healthy food". "I felt happy to change the choice of food so my life can be healthier."

\section{When iCrave did not work}

We asked participants what their experiences were when iCrave did not help them avoid an unhealthy snack. These experiences appeared markedly different from the moments where iCrave did support healthier choices. Participants spoke of how the app didn't help them and they did not feel any shift in their cravings. E.g., "I felt it could not help me this time." "Nothing changed, both before and after using the app I still wanted chicken." "It didn't work...the feeling was the same both before and after using the app." These remarks suggest that iCrave worked by shifting preferences and mental states, and the situations in which iCrave was ineffective was when this shift did not occur.

\section{DISCUSSION}

\section{Improving snacking in moments of temptation}

Our results showed that engaging in imagery tasks, which have been shown to reduce self-reported craving levels in laboratory studies, can improve snacking behavior when performed at moments of food temptation. Qualitative interview results suggest that the craving-reduction imagery task influenced behavior in several ways. First, it may simply extinguish the desire for unhealthy foods. Second, it may shift preferences away from healthy foods and towards healthier foods. Third, it may reduce the initial desire enough to give users the presence of mind to make a more controlled choice informed by long term health rather than short term desires. Our user-log results showed that reported vividness of the imagery task correlated with moments where healthy snacks and no snacks were chosen, 
supporting the idea that level of engagement in the imagery task was instrumental in influencing whether healthier choices were made.

\section{Beyond momentary benefits}

Our results hint at the possibility that the use of iCrave can provide wider benefits for snacking behavior beyond those moments of using the app. In post-experiment questionnaires, participants who used iCrave reported that they were more able to resist unhealthy snacks compared to those in the tracker condition. While this might have been purely due to the fact that they were using the full iCrave app, another possibility is that by practicing the imagery task, they learned a cognitive strategy for reducing desire, which they could implement even when they were not using the app. While results did not reach significance levels, users of iCrave also reported having overall fewer cravings for unhealthy snacks and being more aware of eating unhealthy snacks in comparison to users of the tracking app. Finally, in our qualitative interviews, some participants mentioned that iCrave prompted greater awareness of food choices even when they were not using the app. Longer term studies will be needed to verify the possible broader effects of using iCrave mentioned above that are suggested by our current work.

\section{Open questions}

Our study focused on the use of iCrave to improve snacking behavior. However, it would also be also interesting to know whether iCrave can improve the healthiness of consumption during meal as well as snacks. Though cravings are typically associated with snacks, they could also play a role in meal time consumption. The observations that iCrave appeared to shift preferences towards healthier foods and help induce a state of mind where one is able to make healthier choices, suggests that it may be useful for meals as well as snacks.

Another line of open enquiry is to understand in more detail the effectiveness of different individual components of iCrave. While our results found that imagery vividness correlated with healthy choices, it is unknown the extent to which a 10s delay without the imagery task might also be effective. Laboratory studies have shown that equivalentlength mind wandering tasks [22] and other cognitive tasks such as reciting the alphabet backwards [25] are not effective in reducing cravings. This suggests that a mere 10 s delay without imagery would not be as effective in changing behavior. However, this remains to be empirically verified.

Another factor that may have contributed to the success of iCrave besides the imagery task and delay was the presence of the 'save' button. While our participants spoke of the imagery task shifting their choice preferences, it is possible that the mere possibility of being able to record moments of overcoming cravings also would be beneficial on its own, without a craving reduction task. Indeed, in user interviews for our lite prototype, which only contained a 'save' button, people reported enjoying recording their savings. However, the effectiveness of this savings button has yet to be systematically evaluated on its own.

\section{Design implications}

Our finding that a system which prompts an imagery task and records moments of overcoming temptation can improve snacking habits opens up questions about how the system might be designed to optimize effectiveness. One possibility is to allow users to engage in multiple repeats of the imagery task and/or to allow the user to select a new imagery task if the first imagery prompt is unappealing. Another option is to allow the user to choose whether they want to have an imagery task at all, thus allowing for more flexibility in usage. Additionally, a greater variety of craving reduction tasks can be incorporated to prevent adaptation. Previous laboratory studies have shown that other imagery tasks such as staring passively at dynamic black and white visual noise, olfactory imagery, and tactilevisual manipulation can also reduce cravings [18-21]. Other studies have shown that imagining indulging in ones favorite activities also can reduce cravings [25]. Thus future versions of iCrave can explore the usefulness of allowing users to create their own repository (e.g., text or photos) of craving reduction material.

Future versions of iCrave can incorporate additional features that enhance the rewards of forgoing snack cravings and reduce the strength of cravings. For example, the application can enhance the association of accumulated savings with future non-food rewards. One can imagine a personal treats store where the user could pre-specify the treats they would allow themselves if they overcame a certain number of cravings or went for a certain time period without unhealthy snacks. Another method for celebrating savings might be to include an option for social features where friends who are trying to snack less are connected together and updates can be posted when each successfully overcomes a craving, allowing others to give congratulations, feedback, or encouragement.

\section{CONCLUSION}

We implemented and tested iCrave, a mobile application designed to influence the specific psychological constructs that surround weight loss: the cost of resisting tempting foods and the rewards of feeling good about having self control. It is the first application to use the cravings reduction methods proposed by EI theory.

We evaluated the effectiveness of our application for improving snacking habits compared to that of a basic snack tracker in a one-week study. We found that while users of both iCrave and the tracker reported eating fewer snacks on average than they usually do, iCrave significantly reduced unhealthy snacking and overall snacking compared to the basic tracker. Users of our application also felt more successful in improving their snack habits, felt more able to 
resist unhealthy snacks compared to users of the simple tracker, reported significantly reduced cravings for snacks overall and reduced cravings for unhealthy snacks compared to before the week of using the application.

Our work demonstrates how a simple mobile app can help individuals overcome snack cravings and improve their snack habits. The design of our application was based on mitigating the discouraging psychological constructs of a desired behavior and enhancing the encouraging ones. iCrave represents one possible instantiation of the many design opportunities afforded through designing to alter the psychological constructs that underlie behavior. The success of our application demonstrates that this is a viable new approach for opening up the design space for persuasive technologies.

\section{ACKNOWLEDGMENTS}

We thank Vicky Cullen for helping us with practice interviews, and all participants for their contributions to the study. This research is partially supported by EPSRC Grant EP/G004560.

\section{REFERENCES}

1. Torning, K. and Kukkonen, H., Persuasive system design: state of the art and future directions. Proceedings of the 4th International Conference on Persuasive Technology, Springer, (2009), 30.

2. Brown, B., Chetty, G., and Harmon, E., A system for students to monitor diet and exercise. Proceedings of CHI(2012), 1807-1812.

3. Consolvo, S., McDonald, D., and Landay, J., Theory-Driven Design Strategies for Technologies that Support Behavior Change in Everyday Life. Proceedings of CHI 2009, ACM Press, (2009), 405414.

4. Consolvo, S., Everitt, K, Smith, I I, and Landay, JA., Design requirements for technologies that encourage physical activity. Proceedings of CHI 2006, ACM Press, (2006), 457-466.

5. Lin, J, Mamykina, L, Lindtner, S, Delajoux, G, and Strub, H, Fish 'n' Steps: Encouraging physical activity with an interactive computer game. UbiComp 2006: Ubiquitous Computing, ACM Press, (2006), 261-278.

6. Consolvo, S., klasnja, P., McDonald, D., Avrahami, D. Froehlich J., LeGrand, L., Libby, R., Mosher, K., and Landay, J., Flowers or a robot army? encouraging awareness and activity with personal, mobile displays. UbiComp 2008, ACM Press, (2008), 54-63.
7. Baumer, E. P. S., Katz, S. J., Freeman, J. E., Adams, P., Gonzales, A. L., Pllak, J. P., Retelny, D., Niederdeppe, J., Olson, C. M., and Gay, G. K, Prescriptive Persuasion and Open-Ended Social Awareness: Expanding the Design Space of Mobile Health. Proceedings of CHI 2012, ACM Press, (2012), 476-484.

8. Brown, Brandon, Chetty, Marshini, Grimes, Andrea, and Harmon, Ellie, Reflecting on health: a system for students to monitor diet and exercise. $\mathrm{CHI}^{\prime} \mathrm{O6}$ extended abstracts on Human factors in computing systems, ACM Press, (2006), 1807-1812.

9. Fehr, E. and Rangel, A., Neuroeconomic Foundations of Economic Choice-Recent Advances. Journal of Economic Perspectives, 25, (2011), 3-30.

10. Oinas-Kukkonen, $\mathrm{H}$ and Harjumaa, M, Persuasive systems design: Key issues, process model, and system features. Communications of the Association for Information Systems, 24, (2009), 28.

11. Oinas-Kukkonen, $\mathrm{H}, \mathrm{A}$ foundation for the study of behavior change support systems. Personal and Ubiquitous Computing, 17, (2013), 1123-1135.

12. Fogg, B. J., Eckles, D., Bogost, I., Consolvo, S., Holmen, E, Spasojevic, M, Ulm, J, Tanguay, S, Walker, S, and White, S, Mobile Persuasion: 20 Perspectives of the Future of Behavior Change, Fogg, B. J. and Eckles, D., Stanford Captology Media,Stanford, 2007.

13. Rachuri, K, Musolesi, M, Mascolo, C, Rentfrow, P, Longworth, C, and Aucinas, A, EmotionSense: A Mobile Phones based Adaptive Platform for Experimental Social Psychology Research. Proceedings of the 12th ACM International Conference on Ubiquitous Computing, ACM Press, (2010),

14. Maitland, J. and Chalmers, M., Designing for Peer Involvement in Weight Management. Proceedings of CHI 2011, ACM Press, (2011), 315-324.

15. Lee, M., Kiesler, S., and Forlizzi, J., Mining Behavioral Economics to Design Persuasive Technology for Healthy Choices. Proceedings of CHI 2011, ACM Press, (2011), 325-334.

16. Maitland, J., Towards Negotiation as a Framework for Health Promoting Technology. ACM SIGHIT Record, 1, (2011), 10-19. 
17. Hsu, A. and Blandford, A., Designing for Psychological Change: Individuals' Reward and Cost Valuations in Weight Management. Journal of Medical Internet Research(2014),

18. May, J., Andrade, J., Batey, H., Berry, L. M., and Kavanagh, D. J., Less food for thought. Impact of attentional instructions on intrusive thoughts about snack foods. Appetite, 55, (2010), 279-287.

19. Andrade, J., Pears, S., May, J., and Kavanagh, D. J., Use of a clay modeling task to reduce chocolate craving. Appetite, 58, (2012), 955-963.

20. Kemps, Eva and Tiggemann, Marika, Modalityspecific imagery reduces cravings for food: an application of the elaborated intrusion theory of desire to food craving. Journal of Experimental Psychology: Applied, 13, (2007), 95.

21. Harvey, Kirsty, Kemps, Eva, and Tiggemann, Marika, The nature of imagery processes underlying food cravings. British Journal of Health Psychology, 10, (2005), 49-56.

22. Hamilton, J, Fawson, S, May, J, Andrade, J, and Kavanagh, D J., Brief guided imagery and body scanning interventions reduce food cravings.

Appetite, 71, (2013), 158-162.
23. May, J., Andrade, J., Panabokke, N., and Kavanagh, D., Images of desire: cognitive models of craving. Memory, 12, (2004), 447-461.

24. May, J., Andrade, J., and Kavanagh, D., Elaborated Intrusion Theory: A cognitive-emotional theory of food craving. Current Obesity Reports, 1, (2012), 114-121.

25. Knauper, B., Pillay, R., Lacaille, J., McCollam, A., and Kelso, E., Replacing craving imagery with alternative pleasant imagery reduces craving intensity. Appetite, 57, (2011), 173-178.

26. Baddeley, A D. and Andrade, J, Working memory and the vividness of imagery. Journal of Experimental Psychology: General, 129, (2000), 126.

27. Liu, Y., Gao, J. H., Liu, H. L., and Fox, P. T., The temporal response of the brain after eating revealed by functional MRI. Nature, 405, (29-6-2000), 10581062.

28. de, Lauzon B., Romon, M., Deschamps, V., Lafay, L., Borys, J. M., Karlsson, J., Ducimetiere, P., and Charles, M. A., The Three-Factor Eating Questionnaire-R18 is able to distinguish among different eating patterns in a general population. J.Nutr., 134, (2004), 2372-2380. 